Proc. of Intern. Conf. 'Frontiers of Fundamental Physics', September 27-30, 1993, Olympia, Greece (Plenum Press, New York 1994) pp.153-162.

\title{
UNIVERSALITY OF THE LIE ISOTOPIC SYMMETRIES FOR DEFORMED MINKOWSKIAN METRICS
}

\author{
A.K. Aringazin and K.M. Aringazin \\ Department of Theoretical Physics \\ Karaganda State University, Karaganda 470074, \\ Kazakhstan \\ and Institute for Basic Research \\ P.O. Box 1577, Palm Harbor, FL 34682, U.S.A.
}

\section{INTRODUCTION}

Lie-isotopic and Lie-admissible theories are based on non-trivial realisation and generalisation of the conventional product and Lie algebra. Various studies are now performed in applying this formalism to metric spaces, gauge theory, classical and quantum mechanics, field theory, and quantum groups. Lie-isotopic construction provides consistent generalisations of Hamiltonian mechanics refered to as Birkhoffian mechanics and Birkhoff-Santilli mechanics.

In metric spaces, the application of the Lie-isotopic approach gives rise to generalisations of Minkowskian space-time called Minkowski-isotopic spacetime. This generalisation can be treated as a deformation of the Minkowski space-time providing both gravitational and non-gravitational effects. In 
the non-gravitational sector, Minkowski-isotopic space-time metric has been studied, and various generalisations of the Minkowski metric, which were proposed in the context of particle physics, are shown to fit the Minkowskiisotopic metric. This is due to a general nature of the Lie-isotopic element, which may depend on parameters characterising geometrically a medium, such as anisotropy, velocity-dependence (Finslerian) characteristics.

Lorentz invariance is replaced by more general Lorentz-isotopic invariance. Particlularly, specific effects of the Lorentz-non-invariance (LNI) models may be all interpreted in the context of Minkowski-isotopic approach. Specifically, these models have been suggested to explain anomalous energy dependence of the life-times and other fundamental parameters of unstable particles which has been indicated to not fit the Einsteinian law, in high energy region (above $10 \mathrm{GeV}$ ). As a conclusion, we can state that the Lieisotopic approach gives a natural anzatz to investigate the LNI effects.

In the absence of gravity, space-time is determined as a smooth flat manifold endowed with the Minkowski metric $\eta_{i j}=\operatorname{diag}(+1,-1,-1,-1)$. Transformation group of the space-time which leaves the space-time interval, $d s^{2}=d x^{i} \eta_{i j} d x^{j}$, invariant is the Lorentz group. The Lorentz symmetry is one of the fundamental symmetries of physical theories and various experiments verify it to a high accuracy. The Lorentz symmetry seems to be exact. Evidently, this is correct when one deals with test particles and ordinary conditions. Hovewer, when, for instance, extended particles or high energies, or unusual physical conditions leading to a new reality are considered conventional formalism fails to describe associated relativistic effects.

Now, there are both theoretical and experimental arguments to treat the proper Lorentz symmetry as an approximate symmetry of physical processes. Various models have been proposed and experimental results are obtained to verify this conjecture.

Blokhintsev[1] and Redei[2] suggested the modification of the conventional relativistic life-time formula for unstable particles, $\tau=\tau_{0} \gamma\left(1+10^{25} \gamma^{2} a_{0}^{2}\right)$, where $a_{0}$ is a "fundamental length", $\left[a_{0}\right]=\mathrm{cm}$. According to the original treatment, the length $a_{0}$ plays an universal role in the sense that all conventional fundamental theories must be modified at the $a_{0}$ distance scale.

Nielsen and Picek[3] have developed the Lorentz-non-invariant (LNI) model based on the "minimally" generalised Minkowski metric $g_{i j}=\eta_{i j}+\chi_{i j}, \chi_{i j}=$ $\operatorname{diag}(\alpha, \alpha / 3, \alpha / 3, \alpha / 3)$, with $\alpha$ being small Lorentz symmetry breaking parameter, which suggested to be tangible on the scale of electroweak unifica- 
tion, $a \sim 1 / M_{W} \sim 10^{-16} \mathrm{~cm}$. The traceless additional tensor $\chi_{i j}$ provides a residual $S O(3)$ symmetry. The model yeilds, in particular, the life-time high-energy formula, $\tau=\tau_{0} \gamma\left(1+4 \alpha \gamma^{2} / 3\right)^{-1}$. From consideration of the $\pi$ - , $\mu$ - , and $K$-meson data they obtained the following estimation of the average of $\alpha,\langle\alpha\rangle=(0.54 \pm 0.17) \times 10^{-3}$. It should be stressed that this LNI model does not lead to $C P$-violating physics[3].

Also, series of $K$-meson regeneration experiments at Fermilab[4], in the energy range $E_{K} \sim 30-130 \mathrm{GeV}$, display an anomalous energy dependence of the fundamental parameters of the $K^{0}-\bar{K}^{0}$ system . Aronson et al. [5] have shown that the eventual anomalous behavior can not be attributed to an electromagnetic or hypercharge field, or to the scattering of kaons from stray charges or cosmological neutrinos, and that it can not arise from gravitational interaction either. They arrived at the conclusion that the anomalous energy dependence of the $K^{0}-\bar{K}^{0}$ parameters may be the signature of a new interaction. In course of the work, they supposed that it is due to an interaction of the $K^{0}-\bar{K}^{0}$ system with an external field or medium. To describe the anomalous behavior, Aronson et al.[5] have made the modification of the quantum equation of proper time evolution of the $K^{0}-\bar{K}^{0}$ system by adding to it velocity-dependent terms and, after ultrarelativistic expansion, obtained the values of the slope parameters, $b_{x}^{(N)}$, defined by the following formula: $x=x_{0}\left(1+b_{x}^{(N)} \gamma^{N}\right), \gamma=E_{K} / m, N=1,2$. Here, $x$ denotes the life-time $\tau_{S}$, the mass difference $\Delta m=m_{L}-m_{S}$, and the $C P$-violating parameters $\left|\eta_{ \pm}\right|$and $\tan \phi_{ \pm}$. However, it was emphasised that this treatment is purely phenomenological in that it makes no assumption concerning the origin of the additional velocity-dependent terms. An attemption to treat the origin of such terms on the basis of Lie-isotopic Finslerian lifting of the Lorentz group has been made in [6]. We will discuss this problem below.

Also, in a recent paper by Cardone, Mignani and Santilli[7], the $K_{S}^{0}$ lifetime data reported in [5] have been re-analysed, within the framework of Lie-isotopic approach. They argued that a non-linear dependence of the lifetime on energy is needed, in the energy range above $10 \mathrm{GeV}$, rather than the linear one provided by conventional special relativity. The fit parameter is found to be approximately constant at intermediate energies, 30-100 GeV. Another set of data on the $K_{S}^{0}$ life-time, in the energy range $100-350 \mathrm{GeV}$, reported by Grossman et al.[8] shows no evidence for an energy dependence of the life-time that is in contradiction with the data reported by Aronson et al.[4], in the range 100-130 GeV. So, more experiments are needed to solve 
this contradiction.

Gasperini[9] has considered ultrarelativistic particle motion in the model with broken local Lorentz gauge symmetry. In a cosmological aspect, the broken Lorentz symmetry in the very early universe has been considered[10]. The possibility that the Lorentz non-invariance can be considered as an effect produced by strong gravity has been shown[11]. Also, various gravitational consequences of the theory with broken local Lorentz symmetry were studied[12]. As to a Lie-admissible formalism, Gasperini[13, 14] formulated a Lie-admissible (Lie-isotopic) theory of gravity using the extended geometrical framework based on Lie-admissible (Lie-isotopic) underlying algebra.

Also, Ellis et al.[15] and Zee[16] have discussed the possibility that the proton decay may violate Lorentz invariance in the context of grand unified theories. Zee[16] mentioned, particularly, that in such a small region as an interior of the proton "anything can happen" and that perhaps it is not totally far fetched and outregious idea to allow violation of the Lorentz symmetry at some distance scale, for example, on the scale of superheavy $X, Y$-bosons, $a \sim 1 / M_{G U T} \sim 10^{-23} \mathrm{~cm}$. Lorentz non-invariance of the primodial fluid was suggested by Rosen[17].

For a review of gravitational consequences of an eventual local Lorentz non-invariance and Finslerian approach to relativity and gravitation providing naturally a velocity-dependence framework see[18, 19] . For a comprehensive introduction to Finsler geometry, we refer the reader to monographes by Rund[20], Asanov[21], Matsumoto[22], and Asanov and Ponomarenko[23]. Also, for non-metric effects in flat space-time see[24] and references therein.

Original investigations by Santilli on the Lie-isotopic generalisation of Galilei's and Einstein's relativities has been presented in his monographes[25, 26]. Mathematical foundations of the Lie-isotopic generalisation has been reviewed in a monograph by Kadeisvili[27]. For a comprehensive review of the Santilli's Lie-isotopic generalisation of the relativities we refer the reader to a recent monograph by Aringazin, Jannussis, Lopez, Nishioka, and Veljanoski[28]; see also [29].

In the next section, we present a brief introduction to the Lie-isotopic generalisation of the Lorentz symmetry with special reference to its continuous part.

We discuss in detail problem of internal conditions. Non-Minkowskian part of the metric is assumed to describe geometrically local physical properties of the space-time such as nonhomogeneity, deformations, resistance, 
anisotropy, and velocity-dependence.

The origin of these properties in a microscopic region is perhaps due to some quantum effects, which can lead to small deviations from the conventional pseudo-Euclidean structure in four dimensions, and may be revealed at high energies. It is worthwhile to note here that de Brogle in his theory of double solution emphasised that even if a particle is not subjected to any gravitational or electromagnetic field, its possible trajectories are the same as if space-time possessed the non-Euclidean metrics. So, in view of this theory, the deviations may be sought to be caused by subquantum vacuum fluctuations. However, we shall not discuss this deep issue here.

Discussion in the last section serves both to illustrate more general arguments, and to set the stage for a subsequent description of the Lorentznon-invariance effects within the framework of strict classical algebraic Lieisotopic generalisation.

\section{LORENTZ-ISOTOPIC SYMMETRY}

Lie-isotopic approach in connection with the Lorentz symmetry generalisation problem has been originated by Santilli[30, 31]. Let us recall main aspects of the Lie-isotopic generalisation of the Lorentz transformations.

The associative enveloping algebra $E$ equipped with associative product, $A B$, and the unit $I$ can be isotopically lifted to the algebra $\hat{E}$ with the generalised product $A * B=A T B$ and the new unit $\hat{I}=T^{-1}$, where $T$ is a fixed nonsingular element of $E$. The isotopic lifting of the Lie transformation group $G$ then reads, $\hat{G}: x^{\prime}=g * x=\exp (X u)_{\mid \hat{E}} * x=\exp (X T u)_{\mid E} x$, where $X$ denote generators of the original Lie group $G$ and $u$ are parameters. The isotopic lifting of commutator is defined accordingly by

$$
\left[X_{i}, X_{j}\right]^{*}=X_{i} * X_{j}-X_{i} * X_{j}=\hat{c}_{i j}^{k} * X_{k}
$$

where $\hat{c}=c * \hat{I}$ are structural constants.

Let $E(4, \eta, R)$ be Minkowski space-time. Define isotopic lifting of the Minkowski metric

$$
\eta \rightarrow g=\hat{\eta}(t, x, \dot{x}, \ldots) .
$$

According to Theorem 1 of [30], the isotopic lifting of the Lie group of transformation of the Minkowski-isotopic space $E(4, g, R)$ leaves invariant the metrical form defined by $x * x=x^{i} g_{i j} x^{j}$. Thus, when the isotopic element $T$ is given by the new metric $g$, the new unit is $\hat{I}=g^{-1}$. 
Then, by suitable generalisation of the Minkowski metric (2) providing preservation, under the lifting, connectivity properties of the proper Lorentz group one can construct step by step the isotopic liftings of the enveloping algebra of the Lorentz group, the Lorentz group, and the Lie algebra of the Lorentz group. In the limit

$$
\hat{I} \rightarrow I \quad \text { or } \quad g \rightarrow \eta
$$

Lie-isotopic theory covers conventional one.

Lorentz-isotopic transformations can then be explicitly computed when the new metric $g$ is defined. We note here that in spite of the local isomorphism between the lifted Lorentz group and proper Lorentz group the Lorentz-isotopic transformations may be different from the conventional ones. Clearly, it is worthwhile investigate implications of this generalisation in particle physics. For more complete and precise development of the Lie-isotopy on metric spaces, we refer the reader to $[30,31]$.

As to examples, explicit calculations by Santilli[30] for locally deformed Minkowski metric

$$
x * x=x^{i} g_{i j} x^{j}=x^{1} b_{1}^{2} x^{1}+x^{2} b_{2}^{2} x^{2}+x^{3} b_{3}^{2} x^{3}+x^{4} c^{2} x^{4}
$$

where $b=b(t, x, \dot{x}, \ldots), c=c(t, x, \dot{x}, \ldots)$, have been made, and the associated Lorentz-isotopic transformations found to be in the form

$$
t^{\prime}=\hat{\gamma}\left(t-V b_{3}^{2} z / c\right), \quad z^{\prime}=\hat{\gamma}(z-V t), \quad \hat{\gamma}=\left(1-V b_{3}^{2} V / c^{2}\right)^{-1 / 2}
$$

The metric (4) with the associated generalised Lorentz transformations (5) have been used in $[6,7]$ to describe anomalous energy dependence of the parameters of the $K^{0}-\bar{K}^{0}$ system[4].

Another example is given by anisotropic Finslerian metric by Bogoslovski[32]

$$
x * x=x^{i} g_{i j} x^{j}=x^{i}\left[\left(\frac{\nu^{k} \eta_{k l} \nu^{l}}{x^{k} \eta_{k l} x^{l}}\right)^{r / 2} \eta_{i j}\right] x^{j}
$$

where $\nu^{i}$ is an anisotropy vector, and $r$ is a scale parameter. Associated generalised Lorentz transformations are

$$
t^{\prime}=\hat{\gamma}(t-V z / c), \quad z^{\prime}=\hat{\gamma}(z-V t), \quad \hat{\gamma}=\left[\frac{1-V / c}{1+V / c}\right]^{r / 2}\left(1-\frac{V^{2}}{c^{2}}\right)^{-1 / 2}
$$




\section{LIE-ISOTOPIC LIFTING}

Examples presented in previous Section show that the Lie-isotopy provides a useful method to derive generalised Lorentz transformations while in the proper geometrical approach, for example, within Finslerian framework, one meets difficulties in constructing an explicit form of generalised Lorentz transformations leaving generalised metric form invariant (see, e.g., discussions in $[19,21])$.

Note that there are no theoretical constraints for dependence of the metric $g$ on local variables except for general demands such as non-singularity. So, the question how one can determine the non-Minkowskian dependence of $g$ for a specific application arises. Equivalently, the question is how one can choose specific element $T$ of the isotopic class of generalisation. Fixing the isotopic element $T$ of the algebra $E$ means that one choose fixed generalised metric and, accordingly, fixed type of generalised Lorentz transformations associated to this algebra.

\section{Phenomenological approaches}

One way to pick out isotopic element $T$ among possible ones is to suppose that the generalised metric should be derived uniquely from given internal physical conditions. Conventional theory and experiments of special relativity learns us that the ordinary conditions, i.e., the case of "empty" space-time and point-like particles, should lead to conventional Minkowski metric. If this is not the case - complicated internal conditions - one can appropriately generalise the metric, and then derive associated generalised Lorentz transformations.

Washing out such complicated conditions should yield contunious reducing of the isotopic unit, $\hat{I}=g^{-1}$, to the ordinary one due to (3). This obvious requirement put strong limits on possible generalisations and does not admite exotic metric structures.

However, in general case a way to derive the generalised metric from (suppose known) internal conditions is far from being strightforward. One can try axiomatic way defining some specific type of the metric a priori, and then verifying the associated Lorentz-isotopic symmetry.

Once more way to specify the metric $g$ is to account for the internal Lorentz-non-invariance effects on a phenomenological level. This method, 
albeit in somewhat ad hoc fashion, enables one to apply the generalised theory when internal conditions are not presented by well defined (Lagrangianbased) equations for the metric $g$.

It is highly remarkable that any phenomenological deviation from the Minkowski metric finds its counterpart in the Lie-isotopic formalism so that all such deviations can be treated, in effect, as the Lie-isotopic extension of the conventional underlying algebra.

In terms of small deviations from conventional Lorentz symmetry, one can proceed as follows. General expansion of the metric $g$ on the Minkowskian background can be performed to set a parametrical representation of the lowest approximation. Then, one need to establish for what physical effects the parameters entering the metric are responsible. The sizes of the effects, if measured, will put upper limits or estimations on these parameters. Known example is the simplest LNI parametrisation by Nielsen and Picek[3] mentioned in Introduction where meson life-times data had been used to estimate value of the LNI parameter $\alpha$. Another example[6] concerns to an ultrarelativistic expansion of the velocity-dependent metric (4), with estimations on $\lambda^{\prime}$ 's in series, $b_{3}^{2}=1+\lambda_{0}+\lambda_{1} \gamma+\lambda_{2} \gamma^{2}+\ldots$, being derived from data of the anomalous energy behavior of the $K^{0}-\bar{K}^{0}$ system parameters[4, 5]. In a recent paper by Cardone et al.[7], these data were used to estimate the fit parameter $a=b_{3}^{2} / b_{4}^{2}$.

As it was stressed[7, 30], the LNI parameters of the metric may vary not only from weak interaction to strong one but also from reaction to reaction within each type of interactions. In fact, analysis on meson life-times data[3] showed that the LNI parameter $\alpha$ even has different sign for different meson decays. Thus, dependence of $g$ on parameters is not universal, and should be varied when different physical conditions occur.

We should note that this LNI anzatz is in close analogy with the one of the parametrised post-Newtonian (PPN) framework where all possible self-consistent metrical theories of gravity can be tested on the basis of the "fiducial" PPN metric with experimentally determined PPN parameters (see, for a review, [19, 33]).

Using the metric $g$ one can also try to construct Lagrangian of the theory and then derive equations for internal terms which will impose restrictions on $g$ in a dynamical way. As an example, we refer to the Finslerian theory $[19,21]$ where dependence of the Finslerian metric tensor $g_{i j}(x, y)$ on the "internal" tangent vector $y \in T M$ can be determined from equations for curvature 
tensor of the tangent bundle $T M$. It should be noted that at the same time basic manifold $M$ is characterised by its own curvature tensor, and can be taken flat in the absence of gravity. In a physical context, this means that we have a locally curved momenta space that implies a non-quadratic relation between energy and momentum of a free particle (such a relation arising from supposing that space-time is really a lattice has been discussed by Aronson et al.[5]; see also [14]). On the other hand, one can define a section $\sigma: M \rightarrow T M$ to treate vector $y$ as an auxiliary vector field on $M$ due to the concept of oscullation[21].

In the context of strict geometrical framework, the intrinsic behavior of the internal variable $y$ causing some microscopic non-linear effects has been considered in detail by Ikeda[34]. In particular, Ikeda established that the intrinsic behavior of $y$ is reflected in a whole spatial structure. This observation may have a direct use in the case of algebraic extension of the metrical structure provided by the Lie-isotopic approach.

Also, in Kaluza-Klein anzatz (see, for example, [35]) internal variables (extra dimensions) are assumed to be curled up to a small compact manifold $C$ to be not visible at low energies. Internal fibers are sought to carry a Lie-group srtucture which defines an internal symmetry of the system at each point of space-time $M_{4}$; see also Weinberg's insight[36] and[37] for a recent development of the standard Kaluza-Klein technique. Despite of the relevance of the usual compactification scheme to get a stable vacuum solution for which a vielbein and connections obey the isometry on $M_{4} \times C$ it would be very instructive to construct a dynamical framework for compactification (see discussion in [14]). Also, recent development of superstring theory $[38,39,40]$ revealed that the compactification to Calabi-Yau manifold reducing ten-dimensional theory to four-dimensional one can be provided in enormous numbers of ways. This indicates a non-triviality of the internal condition problem on a fundamental level.

\section{Path integral approach}

Another way to solve the problem of the isotopic metrical degrees of freedom is to require something like an invariance under the Lie-isotopic lifting. Namely, one can treate a set of all Minkowski-isotopic metrics $g$ as a functional space $H, g \in H$, and introduce a covariant measure in $H$. The theory then will contain integral, $S=\int d g F[g]$, over all possible Minkowski-isotopic 
metrics defined on a manifold with fixed topology. To define volume element in the space of all metrics $g$ one first need to define the "distance" $\|g\|$ between infinitesimally different metrics, $g$ and $g+\delta g$. The metric variation $\delta g$ can be appropriately parametrised to adapt symmetries of the theory. For example, general coordinate transformation parameters, rotational transformation parameters of the internal vector $y$, and the conformal transformation parameter $\sigma, g_{i j}(x, y) \rightarrow \exp [2 \sigma(x, y)] g_{i j}(x, y)$, can collectively constitute possible set of variables, $u^{a}$, in respect of which the variation $\delta g$ can be formulated. The measure will be of the following bilinear form:

$$
\|\delta g\|^{2}=P_{a b} \delta u^{a} \delta u^{b}
$$

So, the integration will be made over these independent parameters $u^{a}$,

$$
S=\int d u \sqrt{\operatorname{Det}(P)} F[g]
$$

We refer the reader to [40] to consult for measures in space of metrics in the context of string theories. It should be noted that the rotational invariance does not eliminate the dependence of the metric on $y$ so that the generalised character of the associated Lorentz-isotopic transformations still remains in this case.

Another choice is to require complete isotopic invariance of the theory under certain class of transformations of the isotopic unit, $\hat{I} \rightarrow \hat{I}^{\prime}$, which includes conventional Minkowski one, $\hat{I} \rightarrow I$. Then, all the Lorentz-isotopic transformations will be equivalent to the proper Lorentz one so that the theory can be formulated in the usual terms, with the only difference of a volume element of $H$ in the action. Introducing of dependence of $g$ on local coordinates $x^{i}$ leads one to consideration of the Lie-isotopic theory of gravity $[13,14,26,28]$, which appears to be more general than general relativity because of the manifested local Lorentz-isotopic symmetry instead of the conventional Lorentz one. However, we do not elaborate further on this topic here restricting our consideration by non-gravitational sector of the Lie-isotopic lifting of the Lorentz symmetry. So, dependence on coordinates $x^{i}$ in the intergral (9) may be treated as a formal one, and one can omit it to avoid additional complications arising in part from topology of basic manifold $M$. We wish to note, incidentally, that the Lie-isotopic gravity, especially in view of the proposed invariance in respect to Lie-isotopic lifting, may be of 
interest in low dimensions, $2+1$ or $1+1$, where one deals with Riemannian surfaces[38, 39, 40, 41].

Perhaps, these suggestions seem to be speculative at this stage, and its realisation will be complicated. However, it can be considered as a very attractive alternative way which enables us to exploit the idea of a symmetry in the space of Lie-isotopically lifted metrics, on a high-energy scale.

\section{CONCLUDING REMARKS}

Although we have phrased our discussion in the context of LNI effects, the Lie-isotopic lifting of the Lorentz group may have use as a framework to generalise other fundamental theories such as quantum field theory and quantum gravity. It should be noted also that the Lie-isotopic lifting of a continuous group of transformations provides a Lie-isotopic generalisation of conventional gauge theories[42] which seems to have far reaching implications. Further generalisation of the Lie-isotopic theory is a Lie-admissible approach, within which one can, particularly, describe an evolution of quantum group systems[43], and we refer the interested reader to [44] for a review on Lieadmissible theory and its applications.

\section{References}

[1] D.I.Blokhintsev, Phys. Lett. 12: 272 (1964).

[2] L.B.Redei, Phys. Rev. 145: 999 (1966).

[3] H.B.Nielsen and I.Picek, Phys. Lett. B144: 141 (1982).

[4] B.H.Aronson, G.J.Bock, H-Y.Cheng, and E.Fischbach, Phys. Rev. D28: 476 (1983).

[5] B.H.Aronson, G.J.Bock, H-Y.Cheng, and E.Fischbach, Phys. Rev. D28: 495 (1983).

[6] A.K.Aringazin, Hadronic J. 12: 71 (1989).

[7] F.Cardone, R.Mignani, and R.M.Santilli, J. Phys. G18: L61 (1992).

[8] N.Grossman et al., Phys. Rev. Lett. 59: 18 (1987). 
[9] M.Gasperini, Phys. Lett. B177: 51 (1986).

[10] M.Gasperini, Phys. Rev. D33: 3594 (1986); Phys. Lett. B163: 84 (1985).

[11] M.Gasperini, Phys. Lett. B141: 364 (1984).

[12] M.Gasperini, Phys. Rev. D33: 3594 (1986); Mod. Phys. Lett. A2: 385 (1987); Class. Quantum Grav. 4: 485 (1987).

[13] M.Gasperini, Nuovo Cimento B81: 7 (1984); Hadronic J. 7: 234, 650, 971 (1984).

[14] M.Gasperini, Nuovo Cimento A83: 309 (1984).

[15] J.Ellis, M.Gaillard, D.Nanopoulos, and S.Rudaz, Nucl. Phys. B176: 61 (1980).

[16] A.Zee, Phys. Rev. D25: 1864 (1982).

[17] N.Rosen, Astrophys. J. 297: 347 (1985).

[18] A.K.Aringazin and G.S.Asanov, Gen. Rel. Grav. 17: 1153 (1985).

[19] A.K.Aringazin and G.S.Asanov, Rep. Math. Phys. 25: 183 (1988).

[20] H.Rund, "The Differential Geometry of Finsler Spaces," Springer, Berlin (1959).

[21] G.S.Asanov, "Finsler Geometry, Relativity and Gauge Theories," D.Reidel, Dordrecht (1985).

[22] M.Matsumoto, "Foundations of Finsler Geometry and Special Finsler Spaces," Kaiseisha Press, Kaiseisha (1986).

[23] G.S.Asanov and S.P.Ponomarenko, "Finsler Fiber Bundle Over Space-Time, Associated Gauge Fields and Connections," Stiinca, Kishinev (1989).

[24] A.K.Aringazin and A.L.Mikhailov, Class. and Quantum Grav. 8: 1685 (1991).

[25] R.M.Santilli, "Foundations of Theoretical Mechanics," Vol.II, Springer, New York (1983).

[26] R.M.Santilli, "Isotopic Generalization of Galilei's and Einstein's Relativities," Vols. I,II, Hadronic Press, Palm Harbor (1991).

[27] J.Kadeisvili, "Santilli's Isotopies of Contemporary Algebras, Geometries and Relativities," Hadronic Press, Palm Harbor (1992). 
[28] A.K.Aringazin, A.Jannussis, D.F.Lopez, M.Nishioka, and B.Veljanoski, "Santilli's Lie-Isotopic Generalization of Galilei's and Einstein's Relativities," Kostarakis Publishers, Athens (1991).

[29] A.K.Aringazin, A.Jannussis, D.F.Lopez, M.Nishioka, and B.Veljanoski, Algebras, Groups and Geometries 7: 211 (1990).

[30] R.M.Santilli, Lett. Nuovo Cimento 37: 545 (1983).

[31] R.M.Santilli, Hadronic J. 8: 25, 36 (1985).

[32] G.Yu.Bogoslovski, Nuovo Cimento B40: 116 (1977); B43: 377 (1978).

[33] C.M.Will, "Theory and Experiment in Gravitational Physics," Cambridge Univ. Press, London (1981).

[34] S.Ikeda, J. Math. Phys. 26: 958 (1985); Nuovo Cimento B100: 493 (1987).

[35] E.Witten, Nucl. Phys. B186: 412 (1981); S.Randjbar-Daemi, A.Salam, and J.Strathdee, Nucl. Phys. B214: 491 (1983).

[36] S.Weinberg, Phys. Lett. B125: 265 (1983).

[37] M.Chaichian, A.P.Demichev, and N.F.Nelipa, Phys. Lett. B169: 327 (1986).

[38] M.B.Green, J.H.Schwarz, and E.Witten, "Superstring Theory," Cambridge Univ. Press, Cambridge (1987).

[39] W.Siegel, "Introduction to String Theory," World Sci., Singapore (1988).

[40] A.M.Polyakov, "Gauge Fields and Strings," Harwood Academic Publ., Chur (1987).

[41] E.Witten, Preprint IASSNS-HEP-88/32 (1988).

[42] M.Gasperini, Hadronic J. 6: 935, 1462 (1983); M.Nishioka, Hadronic J. 7: 1636 (1984).

[43] A.Jannussis, G.Brodimas, and R.Mignani, J. Phys. A24: L775 (1991).

[44] "Proc. of the Third Workshop on Lie-Admissible Formulations," Hadronic Press, Nonantum (1981); "Proc. of the First Intern. Confer. on Non-Potential Interactions and Their Lie-Admissible Treatment," Hadronic Press, Nonantum (1982). 